\title{
Correction to: Medication overuse and drug addiction: a narrative review from addiction perspective
}

Tatiane Teru Takahashi ${ }^{1{ }^{2 *}{ }^{*}}$, Raffaele Ornello ${ }^{3+}$, Giuseppe Quatrosi ${ }^{4}$, Angelo Torrente ${ }^{5}$, Maria Albanese ${ }^{6}$, Simone Vigneri ${ }^{7,8}$, Martina Guglielmetti ${ }^{9,10}$, Cristiano Maria De Marco ${ }^{9}$, Camille Dutordoir ${ }^{11}$, Enrico Colangeli ${ }^{2}$, Matteo Fuccaro ${ }^{12}$, Davide Di Lenola ${ }^{13}$, Valerio Spuntarelli ${ }^{14}$, Laura Pilati ${ }^{5}$, Salvatore Di Marco ${ }^{5}$, Annelies Van Dycke ${ }^{15}$, Ramla Abuukar Abdullahi ${ }^{1,16}$, Antoinette Maassen van den Brink ${ }^{15}$, Paolo Martelletti ${ }^{9,17}$ and on behalf of the European Headache Federation School of Advanced Studies (EHF-SAS)

Correction to: J Headache Pain 22, 32 (2021) https://doi.org/10.1186/s10194-021-01224-8

Following the publication of the original article [1], we were notified that the correct affiliation for Dr. Annelies Van Dycke is:

Department of Neurology, AZ Sint-Jan Brugge, Ruddershove 10, 8000 Brugge, Belgium

Also the acronym for the European Headache Federation School of Advanced should be EHF-SAS.

The original article has been corrected.

\begin{abstract}
Author details
${ }^{1}$ Headache Research, Wolfson CARD, Institute of Psychiatry, Psychology \& Neuroscience, King's College London, 20 Newcomen St, London SE1 1YR, UK. ${ }^{2}$ Present address: Medicines Discovery Catapult, Block 35, Mereside, Alderley Park, Cheshire SK10 4TG, UK. ${ }^{3}$ Neuroscience Section, Department of Applied Clinical Sciences and Biotechnology, University of L'Aquila, Via Vetoio 1, Coppito, 67100 L'Aquila, Italy. ${ }^{4}$ Department of Health Promotion, Mother and Child Care, Internal Medicine and Medical Specialties (PROMISE), University of Palermo, Piazza delle Cliniche 2, 90127 Palermo, Italy. ${ }^{5}$ Department of Biomedicine, Neurosciences and Diagnostic (Bi.N.D.), University of Palermo, Via del Vespro 129, 90127 Palermo, Italy. 'Department of Systems Medicine, University of Rome "Tor Vergata"; Neurology Unit, "Tor Vergata" Hospital, Viale Oxford, 81, 00133 Rome, Italy. ${ }^{7}$ Department of Experimental
\end{abstract}

Biomedicine and Clinical Neurosciences, University of Palermo, Via del Vespro, 129, 90127 Palermo, Italy. ${ }^{8}$ Pain Medicine Unit, Santa Maria Maddalena Hospital, Occhiobello, Italy. ${ }^{9}$ Regional Headache Referral Center, Sant'Andrea Hospital, Via di Grottarossa 1039, 00189 Rome, Italy.

${ }^{10}$ Department of Medical, Surgical and Experimental Sciences, University of Sassari, Piazza Università, 21, 07100 Sassari, Italy. ${ }^{11}$ Department of Neurology, University Hospital Ghent, Corneel Heymanslaan 10, 9000 Ghent, Belgium. ${ }^{12}$ Department of Neurology, Conegliano Hospital, Via Brigata Bisagno, 2 , 31015 Conegliano, Italy. ${ }^{13}$ Department of Medico-Surgical Sciences and Biotechnologies, "Sapienza" University of Rome, Polo Pontino, Viale XXIV Maggio 7, 04100 Latina, Italy. ${ }^{14}$ Department of Internal Medicine, Division of Pharmacology, Erasmus MC University Medical Centre Rotterdam, Wytemaweg 80, 3015, CN Rotterdam, The Netherlands. ${ }^{15}$ Department of Neurology, AZ Sint-Jan Brugge, Ruddershove 10, 8000 Brugge, Belgium. ${ }^{16}$ Headache Centre, Guy's and St Thomas NHS Trust, London, UK.

${ }^{17}$ Department of Clinical and Molecular Medicine, Sapienza University of Rome, Via di Grottarossa 1039, 00189 Rome, Italy.

Published online: 05 July 2021

\section{Reference}

1. Takahashi (2021) Medication overuse and drug addiction: a narrative review from addiction perspective. J Headache Pain 22:32. https://doi.org/10.1186/ s10194-021-01224-8

* Correspondence: tati.pharmacy@gmail.com

${ }^{\dagger}$ Tatiane Teru Takahashi and Raffaele Ornello contributed equally to this work.

${ }^{1}$ Headache Research, Wolfson CARD, Institute of Psychiatry, Psychology \& Neuroscience, King's College London, 20 Newcomen St, London SE1 1YR, UK ${ }^{2}$ Present address: Medicines Discovery Catapult, Block 35, Mereside, Alderley Park, Cheshire SK10 4TG, UK

Full list of author information is available at the end of the article 